This item was submitted to Loughborough's Research Repository by the author.

Items in Figshare are protected by copyright, with all rights reserved, unless otherwise indicated.

\title{
Robot-enhanced therapy: Development and validation of supervised autonomous robotic system for autism spectrum disorders therapy
}

PLEASE CITE THE PUBLISHED VERSION

PUBLISHER

IEEE

VERSION

AM (Accepted Manuscript)

\section{PUBLISHER STATEMENT}

(C) 2019 IEEE. Personal use of this material is permitted. Permission from IEEE must be obtained for all other uses, in any current or future media, including reprinting/republishing this material for advertising or promotional purposes, creating new collective works, for resale or redistribution to servers or lists, or reuse of any copyrighted component of this work in other works.

\section{LICENCE}

All Rights Reserved

\section{REPOSITORY RECORD}

Cao, Hoang-Long, Pablo G. Esteban, Madeleine Bartlett, Paul Baxter, Tony Belpaeme, Erik Billing, Haibin Cai, et al.. 2019. "Robot-enhanced Therapy: Development and Validation of Supervised Autonomous Robotic System for Autism Spectrum Disorders Therapy”. figshare. https://hdl.handle.net/2134/9955427.v1. 


\title{
Robot-Enhanced Therapy \\ Development and Validation of a Supervised Autonomous Robotic Sys- tem for Autism Spectrum Disorders Therapy
}

\author{
Hoang-Long Cao ${ }^{1 *}$ Pablo G. Esteban ${ }^{1 *}$, Madeleine Bartlett ${ }^{2}$, Paul Baxter ${ }^{3}$, Tony \\ Belpaeme $^{2,4}$, Erik Billing ${ }^{5}$, Haibin Cai $^{6}$, Mark Coeckelbergh ${ }^{7}$, Cristina Costescu, \\ Daniel David ${ }^{8}$, Albert De Beir ${ }^{1}$, Daniel Hernandez ${ }^{2}$, James Kennedy ${ }^{2}$, Honghai Liu ${ }^{9}$, \\ Silviu Matu ${ }^{8}$, Alexandre Mazel ${ }^{10}$, Amit Pandey ${ }^{10}$, Kathleen Richardson ${ }^{7}$, Emmanuel \\ Senft $^{2}$, Serge Thill ${ }^{2}$, Greet Van de Perre ${ }^{1}$, Bram Vanderborght ${ }^{1}$, David Vernon ${ }^{5,11}$, \\ Kutoma Wakunuma ${ }^{7}$, Hui $\mathrm{Yu}^{9}$, Xiaolong Zhou ${ }^{12}$, and Tom Ziemke ${ }^{5,13}$ \\ ${ }^{1}$ Robotics and Multibody Mechanics Research Group and Flanders Make, Vrije Universiteit Brussel, Brussels, Belgium \\ ${ }^{2}$ Centre for Robotics and Neural Systems, Plymouth University, Plymouth, U.K. \\ ${ }^{3}$ Lincoln Centre for Autonomous Systems, School of Computer Science, University of Lincoln, Lincoln, U.K. \\ ${ }^{4}$ IDLab - imec, Ghent University, Ghent, Belgium \\ ${ }^{5}$ Interaction Lab, School of Informatics, University of Skövde, Skövde, Sweden \\ ${ }^{6}$ Department of Computer Science, Loughborough University, UK \\ ${ }^{7}$ Centre for Computing and Social Responsibility, Faculty of Technology, De Montfort University, Leicester, U.K. \\ ${ }^{8}$ Department of Clinical Psychology and Psychotherapy, Babes-Bolyai University, Cluj-Napoca, Romania \\ ${ }^{9}$ School of Computing, University of Portsmouth, Portsmouth, U.K. \\ ${ }^{10}$ Softbank Robotics Europe, Paris, France \\ ${ }^{11}$ Department of Computer and Electrical Engineering, Carnegie Mellon University Africa, Rwanda \\ ${ }^{12}$ College of Computer Science and Technology, Zhejiang University of Technology, China \\ ${ }^{13}$ Human-Centered Systems, Department of Computer \& Information Science, Linköping University, Linköping, Sweden \\ EC-FP7 DREAM Project - www. dream2020. eu
}

$\mathrm{R}$ obot-Assisted Therapy (RAT) has shown potential advantages to improve social skills for children with Autism Spectrum Disorders (ASD). This paper overviews the technology development and clinical results of the EC-FP7 funded DREAM project that aims to develop the next level of RAT in both clinical and technological perspectives - which we term Robot-Enhanced Therapy (RET). Within the project, a supervised autonomous robotic system is collaboratively developed by an interdisciplinary consortium, including psychotherapists, cognitive scientists, roboticists, computer scientists and ethicists, allowing the robot control to go beyond the classical remote control methods (Wizard of $\mathrm{Oz}-\mathrm{WoZ}$ ) while ensuring safe and ethical robot behavior. Rigorous clinical studies are conducted to validate the efficacy of RET. Current results indicate that RET can obtain equivalent performance compared to human

${ }^{*}$ H. -L. Cao and P. G. Esteban contributed equally to this work. standard therapy for children with ASD. We also discuss the next steps of developing RET robotic systems.

\section{Towards Robot-Enhanced Therapy}

Autism Spectrum Disorders (ASD) are identified by widespread abnormalities in social interactions and communication together with restricted interests and repetitive behavior [1]. For children with ASD, these symptoms can be efficiently reduced through early (cognitive-)behavioral intervention programs ideally starting at the preschool age [2]. This type of intervention is taught on a one-to-one basis in school and/or at home by caregivers (therapists, teachers, parents) and need to be both intensive and extensive $[2,3]$. This process requires a significant amount of human workload to carry out therapeutic sessions as well as to manage child's performance data.

The use of robots in autism therapy has received 

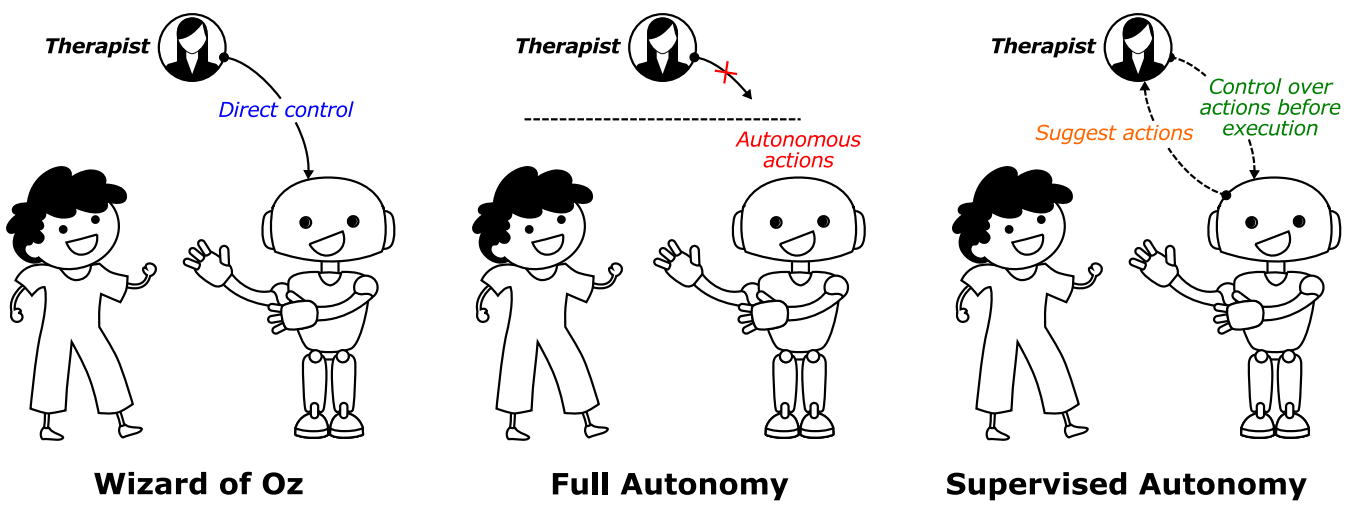

Figure 1: Different robot control paradigms: Wizard of Oz, full autonomy, and supervised autonomy.

attention over the past two decades, with a significant increase in the past one [4]. Similar to animals and computers, robots can provide simple and predictable interactions, in which people with ASD generally feel comfortable, but with several advantages compared to classical therapies [5], as repeatability of the medium's behavior, the embodiment of the medium and the hygienic safety. Robot-Assisted Therapy (RAT) enables embodied interactions that are appealing for many children with ASD, increasing their engagement and attention, and decreasing social anxiety [6]. During child-robot interaction, RAT robots can simultaneously provide social cues while maintaining simplicity and predictability [7]. These robots are diverse in appearance from mobile platforms to humanoid robots. Although RAT robots have shown advantages, most of the studies are exploratory and have methodological limitations [4] such as low participant number or numerous protocol breach.

Regarding the technology development, most of RAT studies are limited to the Wizard of Oz (WoZ) technique in which the robots are remotely controlled, unbeknown to the child, by a human operator (Figure 1-left) $[2,7]$. The WoZ technique allows human therapists to achieve a high level of social interaction without a complex robotic system. However, it requires a significant amount of human workload and is not suitable in the long term [8]. There is a need to increase the level of autonomy of robots in RAT research both to lessen the human workload and to deliver consistent therapeutic experiences [2, 3]. Full autonomy (Figure 1-middle) indicates that the robot makes decisions and adapts its actions to any situation by itself. This is not feasible at this point as the robot's actions must be compliant with the therapeutic goals, the interaction context, and state of the child while its action policies cannot be perfect. Further, fully autonomous robotic systems can raise some critical ethical concerns and are not socially accepted by the general public in the context of interaction with children $[9,10]$. However, a "supervised autonomy" is feasible to achieve in which the robot works autonomously towards achieving given therapeutic goals under a supervisor's guidance (Figure 1-right). When necessary the supervisor can override the robot's actions before execution to ensure that only therapeutically valid actions are executed.

\section{DREAM Project}

The DREAM project aims at implementing RobotEnhanced Therapy (RET) - the next generation of RAT - for children with ASD. This approach calls for the robot's ability to assess a child's behavior by inferring the child's psychological disposition and map the behavior to appropriate actions within specified constraints under supervision of a therapist (i.e. supervised autonomy). The therapist thus is not replaced but rather takes full control of the therapeutic environment with an effective tool and mediator [3].

This paper overviews the technology development and validation of a supervised autonomous robotic system for ASD therapy. The project consortium includes cognitive scientists, roboticist, computer scientists, psychotherapists and ethicists who are collaboratively involved in the development of the system following requirements from different perspectives. The system is validated in a clinical study to assess the effectiveness of socially assistive robots in enhancing social skills i.e. imitation, turn taking, and joint attention. Most importantly, no full-scale randomized clinical trials haven't been carried out in previous research. This has been one of the main goals of the DREAM project. In this project, we also investigate the therapists' attitudes toward the DREAM system and ethical issues related to using (supervised) autonomous robots in ASD therapy.

\section{Requirements for RET systems}

The use of robots in social therapies requires a highly interdisciplinary collaboration. In the DREAM project, all parties (i.e. psychotherapists, engineers, ethicists) have been involved throughout the system development process in a concurrent manner. A robotic system used in RET should meet requirements from both therapeutic and robotic perspectives. Key elements of the 


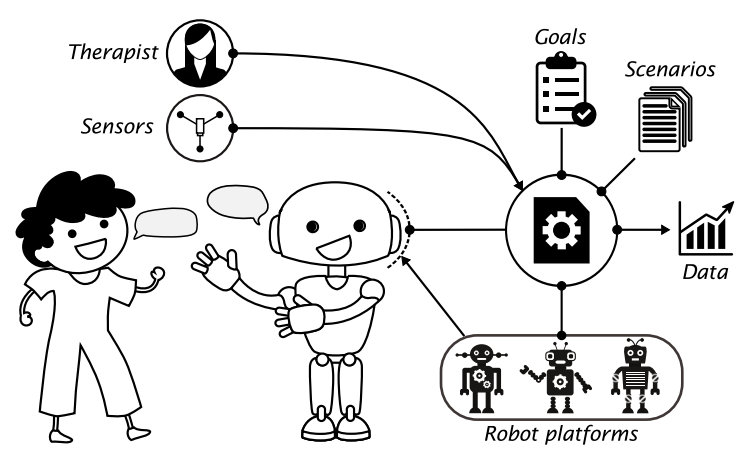

Figure 2: Elements that a RET robotic system should consider to generate robot behaviors [11].

requirements are illustrated in Figure 2 and summarized as follows, see [11].

First, the system should enable the robot to generate task-based and social behavior to achieve therapeutic goals, which is the ultimate goal of using robots in therapeutic contexts. Second, the robot control should be shared with human therapists to ensure safe and ethical behaviors. Third, the system should be applicable to various therapeutic scenarios and robot platforms to reduce engineering workload e.g. reprogramming robot's actions. Lastly, the system should provide and analyze data (e.g. user's performance and performance history, robot operation) recorded in structured forms to different parties.

These requirements serve as guidelines as well as evaluation criteria for RET systems. Some system design principles to obtain these requirements can be: multi-layer behavior organization for generating social and task-based behavior, personalization for providing personalized interaction, and modularity for the ease of applying the system to different scenarios and robot platforms [12]. Under the development of the DREAM project, we have adopted some of these design principles to develop a supervised-autonomous system for different tasks in autism therapy (see Section Supervised-Autonomous System).

\section{Clinical framework}

To assess the socially assistive robots' effectiveness in enhancing social skills in children with ASD, certain behaviors have been frequently targeted by therapeutic interventions. Among them, for the specific goal of the DREAM project, we have targeted the following behaviors: imitation, turn taking, and joint attention. These behaviors could be considered as possible mechanisms underlying the general clinical picture including communication and social interaction deficits, and will be taught by a social robot during repeated therapy sessions of interactive games.

\section{Supervised-Autonomous System}

Controlling a robot to deliver a therapy is a complex task, and in the case of supervised autonomous RET it requires ways to: sense the state and performance of the child, select and execute an action for the robot

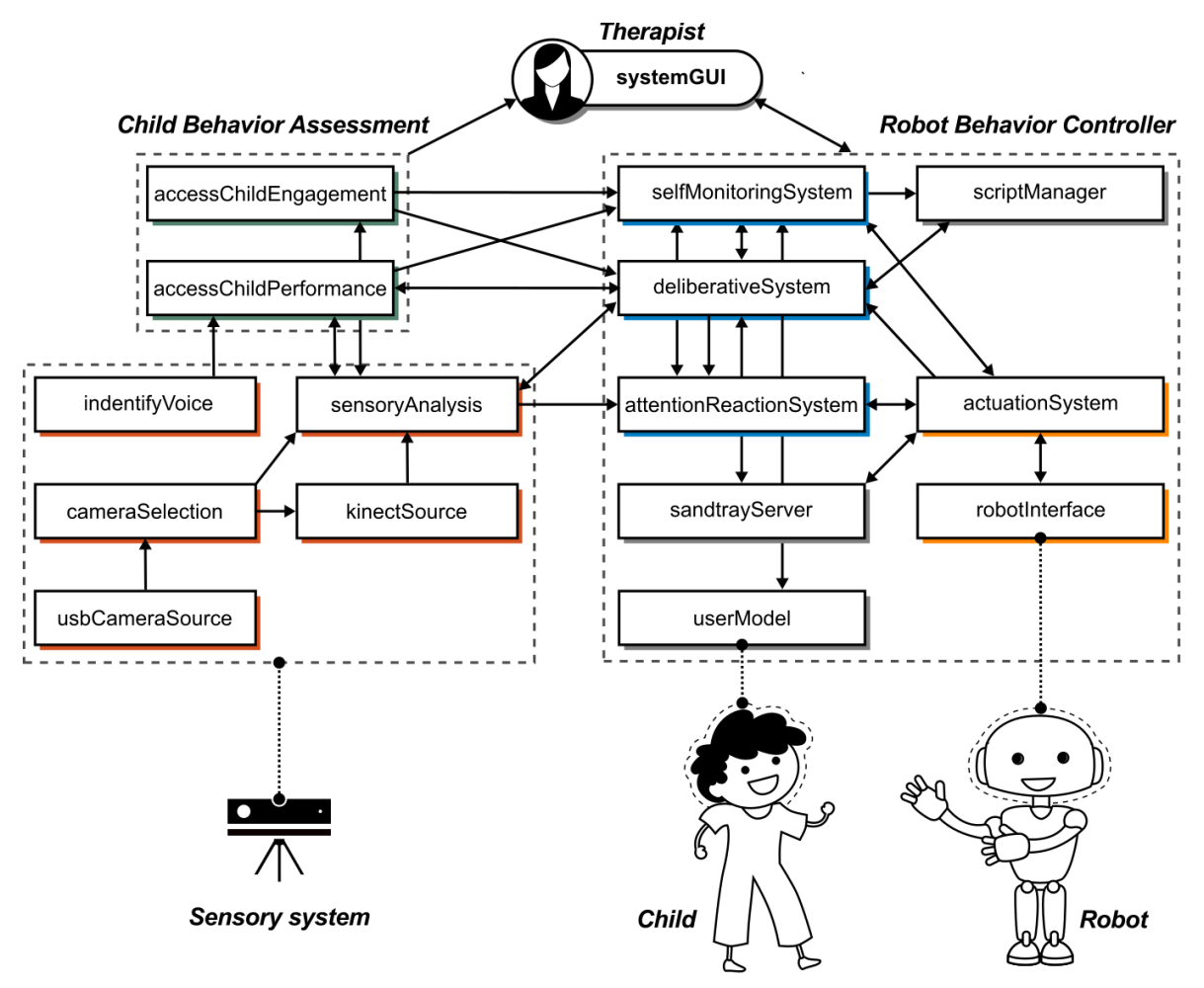

Figure 3: DREAM system architecture. Arrows represent communication between components. 
(according to a therapeutic plan) while providing oversight of the robot's behavior to the therapist. This process is engineered by an interconnected network of components as depicted in Figure 3. These components are responsible for sensing and interpreting the surrounding environment, classifying child behavior and controlling robot behavior. The system also provides an intuitive Graphical User Interface (GUI) allowing the therapist to supervise the system operation and ensure an efficient robot behavior, see Figure 6 . All system components are released ${ }^{1}$ under the GNU GPL v3 license and documented allowing researchers to replicate, modify or expand the DREAM system for different target applications.

\section{Sensory System}

An advanced sensory system interprets multi-sensory data into meaningful information about the interaction between children and the robot e.g. gaze, child's movement, vocal prosody, emotion expression, ASD stereotypical behaviors. Different techniques have been applied on raw images captured by RGB cameras and Microsoft's Kinect sensors for gaze estimation, skeleton joint-based action recognition, face and facial expression recognition, object tracking and audio data processing.

Gaze estimation is significantly important to identify shared attention in child-robot interaction for joint attention tasks. Challenges for gaze estimation that emerge during therapeutic sessions are related to large head movement, illumination variation, and eyelid occlusion. Feature points on the face are located by a Supervised Descent Method based on the best view of the child's frontal face. Head pose is calculated by an object pose estimation method. Iris centers are localized by a hierarchical adaptive convolution method (see red dots in Figure 4a). The final point of gaze is calculated based on the obtained head pose and iris centers by a two-eye model-based method (see the white line in Figure 4a) [13].

Human action recognition, i.e. child's actions during interaction, plays a key role in evaluating the child's performance in imitation tasks. A novel skeleton joint descriptor based on 3D Moving Trend and Geometry property is applied on skeleton data extracted from Kinect depth sensors (Figure 4b) [14]. The descriptor is then used to recognize actions (e.g. waving, touching his/her head with two hands, moving the arms imitating an airplane or covering his/her eyes) by a linear Support Vector Machine (SVM) classification algorithm.

Facial expression recognition provides an understanding of the child's emotion i.e. anger, disgust, fear, happiness, sadness, and surprise. This is achieved by using a frontalization method to recover frontal facial appearances from unconstrained non-frontal facial images

\footnotetext{
${ }^{1}$ https://github.com/dream2020/DREAM/wiki
}

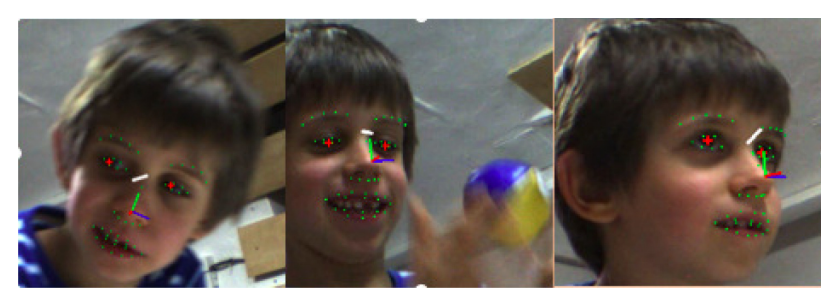

(a) Gaze estimation

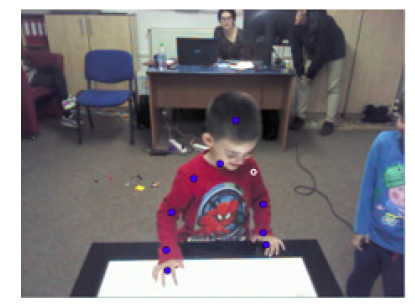

(b) Action recognition

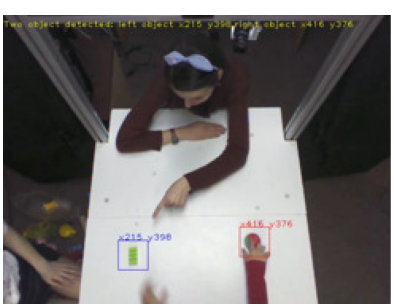

(c) Object tracking
Figure 4: Performance of the advanced sensing system. Images obtained from the interventions.

followed by a Local Binary Patterns feature extraction method on Three Orthogonal Planes to represent facial appearance cues. Finally, we applied an SVM to identify and classify those facial expressions [15] achieving a recognition rate of $63.71 \%$ within real-life conditions. We found quite difficult to achieve a clear partition of emotions as children tend to perform a combination of emotions. However, we obtained better results than other state-of-the-art algorithms.

Object tracking helps to observe the child's behavior regarding the toys on the intervention table, i.e. a plane, a flower, and a cup. A blob based Otsu object detection method is first employed to detect the objects. Then, a Gaussian Mixture Probability Hypothesis Density (GM-PHD) tracker is used to effectively detect and track objects in real time, even when being occluded by hands (Figure 4c) [16].

Audio processing provides information for the robot to perform social attention and evaluate the child's verbal response. Speech recognition and sound direction are based on Kinect SDK. Voices from the therapist and the child are labeled by classifiers such as Gaussian Mixture Model, and Vector Quantification in combination with Mel Frequency Cepstrum Coefficients and Linear Predictive Coding features [17].

\section{Child Behavior Assessment}

Aiming at achieving a supervised autonomous system, the current behavior of the child needs to be appraised. Within this project, this happens in two phases as illustrated in Figure 5.

Data is collected from the Sensory System and mapped onto the identified child behaviors during the first phase. This mapping is based on training and validation sets of child-robots interactions that were previously annotated by knowledgeable therapists. From this process the classifiers provide the probability that 


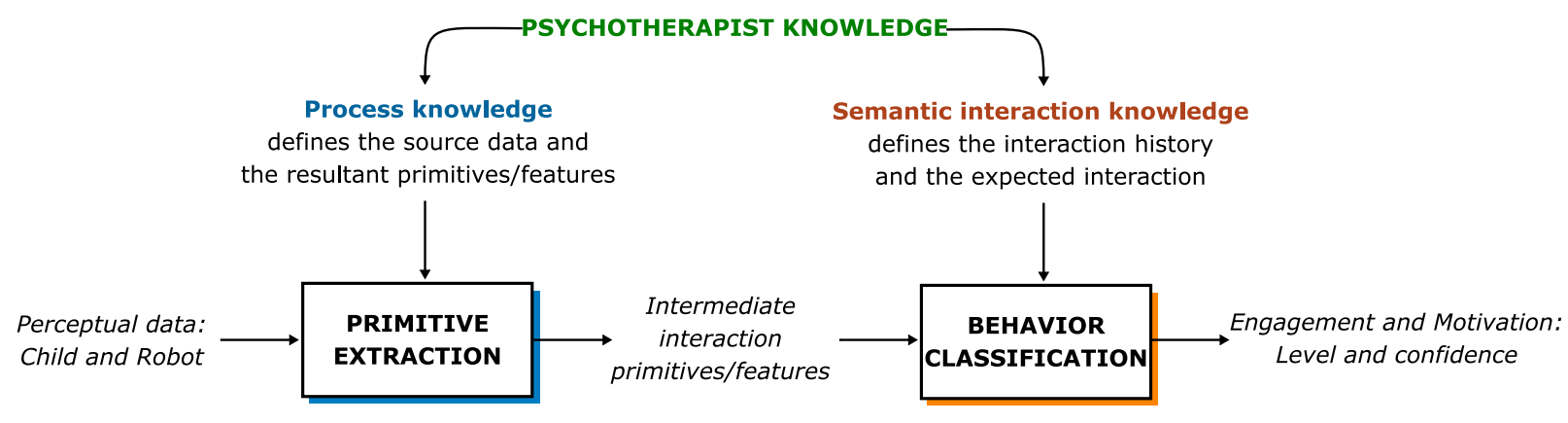

Figure 5: Child behavior analysis flow.

each behavior, among all of them, is currently observed. These probabilities are used into the second phase, where, based on the interaction history, the system attempts to infer the child's level of engagement, motivation and its performance on the task in hand. This second phase relies heavily on the semantic interaction knowledge of the therapists to provide insights into expected patterns.

Classifiers within this system aim at generating real-time annotations of a therapy session as therapists would normally create. Therefore, these autogenerated annotation files might be submitted to therapists for verification and be compared to existing annotations from therapists using standard inter-rater agreement measures. Outcomes of these classifiers are fed into the Robot Behavior Controller allowing the supervised-autonomous operation of the robot.

Additionally, these classifiers might offer other benefits, as being used as a diagnostic tool, relieving therapists of some of their burden. Similar developments have been published but as a binary classification (e.g. non-ASD vs. ASD) [18]. Intermediate degrees of severity of ASD should be accurately identified ranging from "typical of the general population" to "severely atypical". Within the DREAM project, we have started the development of a diagnostic tool based on these classifiers using neuro-computational mechanisms that can be used for learning a large number of dynamical patterns named as conceptors [19].

\section{Robot Behavior Controller}

The robot behavior controller enables the robot to generate task-based and social behaviors, and share the robot control with the human therapist in a supervised autonomous manner. The behavior generation is organized in three layers i.e. attention-reaction, deliberative and self-monitoring, see right side of Figure 3. Behaviors and therapeutic scripts are abstract and non-robot-specific, and later translated into robotspecific motor commands. This allows the system to be platform-independent and scenario-independent. The whole system operation is supervised by a human therapist via a GUI (Figure 6).

The attention-reaction system provides the robot with life-like behaviors e.g. eye blinking, micro-motions,

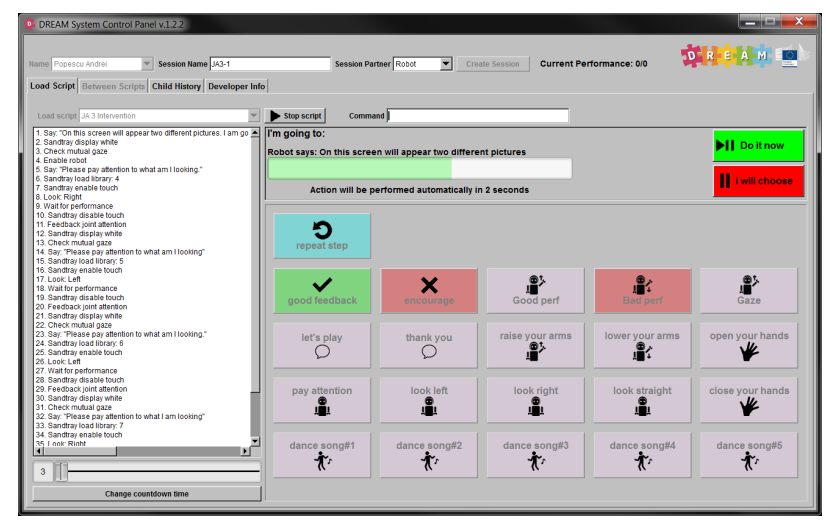

Figure 6: A Graphical User Interface allows human therapists to supervise the system operation.

gaze [20]. These behaviors are essential in social robots. In this system, state information coming from the sensing system is immediately acted upon with appropriate motor outputs. The system also allows the robot to react to the relevant stimulus in the surrounding environment by directing its gaze towards their source. This is achieved by a combination of perceptual and task-related attention, and a target selection algorithm.

The deliberative system is responsible for producing task-based behaviors following therapeutic scripts defined by therapists. These scripts describe step-by-step the high-level desired behaviors of the robot. There are however circumstances that the interaction does not go as planned and the proposed script-based action is not the most appropriate one to perform. For instance, if the child has a low level of engagement with the task, the script following process is paused. The robot seeks for appropriate actions for re-engagement and then returns to the script following process.

In case the action autonomously decided by the robot is not proper, the therapist can deny the suggested action and manually select a more appropriate one. We have proposed a Learning from Demonstration method called SPARC (Supervised Progressively Autonomous Robot Competencies) so that the robot can learn from the manual actions of the therapist and improve its suggested actions next times [21]. As shown in Figure 7, SPARC aims at maintaining high performance 

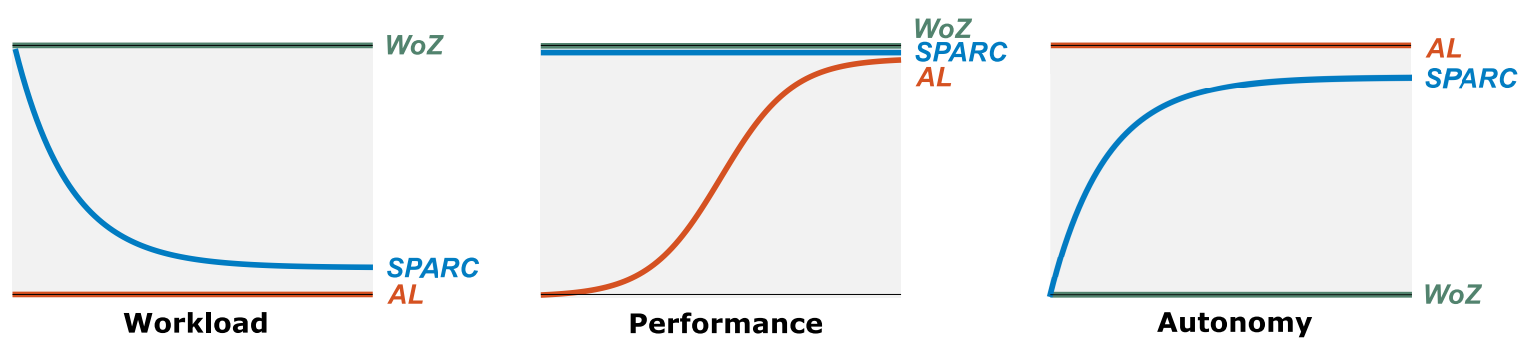

Figure 7: Expected ideal behaviors over time of SPARC in comparison with Wizard-of-Oz (WoZ) and Autonomous Learning (AL) on therapist's workload, robot's performance and autonomy.

throughout the interaction (as in WoZ) while keeping a low therapist's workload (as in Autonomous Learning).

The self-monitoring system aims to overcome possible technical and ethical limitations. The system currently acts as a logging mechanism and is connected with the therapist's supervision interface. The therapist can overrule the robot's proposed actions via the GUI. As future developments, it would act as an alarm system to be triggered when the robot detects technical limitations and ethical issues based on a set of rules. This system also provides recorded data, e.g. child's performance, robot operation, for therapists and engineers to evaluate the efficacy of a RET system.

\section{Clinical experiments and results}

From a clinical perspective, this project seeks to determine how much RET can improve joint attention, imitation and turn-taking skills in ASD children, and how the gains obtained within these interactions compare to standard interventions. Therefore, the clinical experiments were divided into two phases: one using RAT robots under a WoZ system, and another one using RET with a supervised autonomous system. Both phases have been compared to Standard Human Treatment (SHT) conditions.

The experiments were conducted using a classic single-case alternative treatment design. Children participated in six to eight baseline sessions followed by eight SHT sessions and eight WoZ or RET sessions. Within the baseline sessions, the child interacts with a human partner who does not offer any feedback regarding the performance of the child. The purpose of these sessions is to identify the initial level of skills and its variability before the child receives any of the two interventions (SHT or RET, where either the human or robotic partners give feedback that is contingent to the performance of the child).

Conditions were randomized to mitigate the ordering effect. After baseline sessions, the order for each intervention session (either SHT or RAT/RET) was established based on a random schedule which contained a random sequence indicating which session should be performed next. The schedule was different for each child.

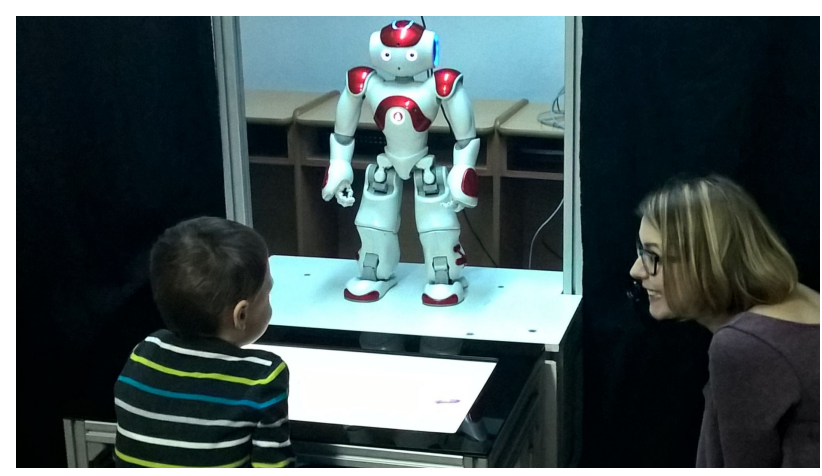

Figure 8: The intervention platform used in DREAM. A child sits in front of a robot and an interactive screen.

Before the baseline session, we used the ADOS instrument [22] to confirm children's diagnosis of autism and to assess which were their social and communication abilities. We also employed ADOS as a measurement tool, to quantify, before and after interventions, the differences in the scores.

Once the initial ADOS measures were taken and the baseline session finished, children interacted with either a robot or a human, always keeping an additional person as a mediator between the child and the interaction partner. The tasks to be tested were implemented following the discrete trial format: highly structured environment, behaviors broken into discrete sub-skills, and child taught to respond to explicit prompting (e.g. "Do like me!").

We have employed the humanoid robot $\mathrm{NAO}^{2}$ to assess our hypothesis. For certain tasks, we used the electronic "Sandtray" [23], a 26-inch capacitive touchscreen and an associated control server, where images can be manipulated by dragging (on the side of the human partner), or simulated dragging (on the side of the robot partner). Moreover, an intervention table was designed to capture sensory information (shown in Figure 8) employing three RGB cameras and two Microsoft Kinect sensors.

Children's performance was assessed in the task by measuring their performance based on task solving accuracy (e.g., accuracy in the imitation task, correct gazing in the joint attention task, appropriate pauses during the turn-taking task).

\footnotetext{
${ }^{2}$ https://www. softbankrobotics.com/emea/en/robots/nao
} 


\section{First phase}

As stated above, within the first phase of the experiments we used RAT robots being remotely controlled under a WoZ setup. Results from these experiments were used as a basis for the development of the Supervised-Autonomous System. For this phase, eleven participants with ASD aged between three and five years old were recruited from the Autism Transylvanian Association.

After completing this first phase, we obtained mixed results. These results were different depending on the task in hand. During the turn-taking task, the WoZ setup seemed to achieve gains as good as or even better than under SHT condition, especially for children with lower levels of prior skills. Regarding joint-attention, RAT and SHT yielded similar outcomes for the majority of the participants. Specific to the RAT intervention, the results have also pointed that the level of prompting offered by the robot mediator has a direct impact on the performance of ASD children, with more prompt increasing the performance [24]. For imitation task, most of the children had already good performances in baseline sessions and the RAT condition did not lead to an improvement in these skills.

\section{Second phase}

In the second phase of the experimental investigations, we are comparing the efficacy of RET and SHT using a randomized clinical trial design ${ }^{3}$. For such purpose, twenty-seven children have been recruited to participate from different organizations and institutions, which provide educational and/or psychotherapeutic services to children with autism, most of them in the city of Cluj-Napoca. Twenty-one have completed the full protocol at the moment.

Results on the Last Observation Carried Forward scores have indicated both groups showed signs of improvement, with a significant time effect, Wilks' Lambda $=.62, F(8,16)=3.19, p=.023, \eta_{p}^{2}=.62$, and no significant group or interaction effects. Univariate analysis indicated that scores have improved for imitation $\left(F(1,25)=21.79, p<.001, \eta_{p}^{2}=.47\right)$ and for each of the turn-taking tasks (sharing information about what one likes, $F(1,25)=4.50, p=.044, \eta_{p}^{2}=.15$; completing a series of figures following a pattern, $F(1,25)=10.22, p=.004, \eta_{p}^{2}=.29$; and categorizing items, $\left.F(1,25)=11.61, p=.002, \eta_{p}^{2}=.32\right)$, but not for joint attention, where baseline differences favoring the SHT group were observed, $F(1,23)=6.66, p=.017$, $\eta_{p}^{2}=.23$. However, post-test differences between groups were not significant for joint attention, even when controlling for baseline scores. In future studies, this outcome will be carefully monitored and more children and sessions might change it in the expected direction. Both interventions had also a positive impact on the clinical ASD symptoms, with children who had

\footnotetext{
${ }^{3}$ ClinicalTrials.gov ID: NCT03323931
}

completed the final assessment reporting lower ADOS severity scores at the end of the treatment in both groups, $t(5)=3.50, p=.017$ in SHT, and $t(4)=3.25$, $p=.031$ in RET.

\section{Therapists' attitudes toward the system}

We conducted an interview to investigate the therapists' attitudes toward the DREAM system. We recruited four therapists that have been working with the system for six months on average. We invited the therapists for an interview via email. During the interview, we used open-ended questions and a short usability survey. A screen-shot of the GUI was used to elicit memories about their experiences with the system.

The DREAM system was generally appreciated by the therapists $(n=4)$. According to the questionnaire results (5-point Likert scale), the therapists showed positive attitudes toward the system i.e. useful $(M=4.1$, Min=4.0, $M a x=4.1)$, satisfying $(M=3.6, \operatorname{Min}=2.4$, $\operatorname{Max}=4.0)$, easy to use $(M=4.2, \operatorname{Min}=4.2, \operatorname{Max}=4.4)$, and easy to learn $(M=3.8, \operatorname{Min}=2.0, \operatorname{Max}=5.0)$. They found that the interface of the GUI is easy to use and helps them deliver an intervention that is both attractive and effective. The automatic detection of the behaviors was useful for the treatment of ASD children as it reduced the burden of the intervention for them as therapists. They also found the system as safe and acceptable. Yet, some improvements would be needed i.e. increasing the accuracy of the recognition, reducing the technical complexity of the system, simplifying of the GUI. Regarding the possibility to use the system for other types of therapies, they suggested having customized GUIs for different applications.

\section{Ethical perspective}

Lately, research in the area of ethics of social robotics has seen significant growth in interest, more specifically in the areas of health-care and children [25].

What are the particular problems raised by autonomous interaction with mentally disabled children? How can we protect children from exploitation? What if the robot gets the behavior assessment of the child wrong? How and when does the therapist need to overrule the behavior of the robot when needed? These questions all raise important ethical concerns. Within the DREAM project, we have conducted several studies to explore this and other ethical issues.

In one of these studies, Coeckelbergh and colleagues aimed at understanding the opinions of parents and therapists about the appropriateness and benefit of social robots being used in therapy for children with ASD [9]. An important finding was the high acceptability of these robots for helping children with autism (85\%). During the study, among the 416 subjects, $22 \%$ were 
parents of children with ASD and 16\% were therapists or teachers of children with ASD. They were surveyed with questions as "Is it ethically acceptable that social robots are used in therapy for children with autism?" or "Is it ethically acceptable to use social robots that replace therapists for teaching skills to children with autism?" This survey indicated the importance of stakeholder involvement in the process with a focus on specific health care issues.

In another study developed within DREAM, Peca and colleagues explored whether age, gender, education, previous experience with robots, or involvement with persons with ASD influences people's attitudes about the use of robots in RET [10]. Results show that these social-demographic factors have a relevant impact on how social robots are perceived e.g. men seem to have a higher level of ethical acceptability compare to women, younger participants seem to be more open to accept the use of social robots in RET for ASD compared to older participants. In terms of the involvement of the participant with children with ASD and the use of social robots in RET, the study show that parents who are not involved directly with ASD children have a higher ethical acceptability level than those who are directly involved.

Finally, Richardson and colleagues have addressed a debate to discuss the risks and challenges of developing research by a multidisciplinary research team with a vulnerable population as children with autism [26]. Given the different backgrounds, research goals, assumptions, and practices, each multidisciplinary research team would approach the research topic from different perspectives: experimental, clinical, engineering, philosophical, and anthropological. Each discipline counts with its own history, terminology, methods and preferences, so that synthesizing these approaches can be challenging.

\section{Discussion and Conclusions}

With the DREAM project, we aimed at implementing Robot-Enhanced Therapy in children with autism interventions. In this paper, we have highlighted the technical development and clinical validation of this approach.

Given the sensitive environment where RET takes place, the DREAM system was developed taking into account requirements from both therapeutic and robotic perspectives, see Section Requirements for RET systems. The supervised-autonomous system follows a multi-layer behavior organization for generating social and task-based behaviors. It was engineered following a modular approach so that, along with being open-source software, the system might be easily used, adapted and/or extended by other research teams to different therapeutic scenarios and robotic platforms.

Our system reaches a performance in par with human therapies commonly used today in clinical stud-

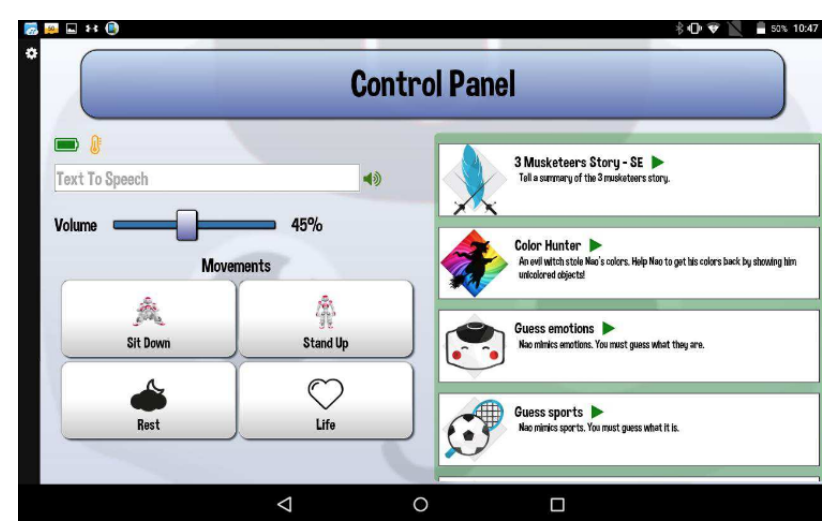

Figure 9: Control panel on teacher tablet.

ies. Despite the mixed results obtained during the single-case experiments, these studies offered valuable insights about the variability of the response of ASD children to RET and pointed to some important issues that should be accounted when developing such interventions (e.g. the need for personalized interventions that match the level of skills of each child). Further exploration needs to be done using variables involved in outcomes such as social engagement; positive and negative emotions; adaptive and maladaptive behaviors; and rational and irrational beliefs [27]. These variables have already been studied for the first phase of the clinical trial. In terms of social engagement, the children showed more interest in the robot partner for the duration of the intervention. Positive emotions appeared more often while interacting with the robot during the imitation and joint attention tasks. The presence of the robot usually acts as a behavioral activator, so that both adaptive and maladaptive behaviors seem to appear more often in the WoZ condition compared to SHT condition. The same study is currently being done for the second phase, and it will offer better answers regarding the relative efficacy of RET and ASD interventions. Future investigations should aim for new research questions beside concerning to whether RET is more or less effective than standard treatment e.g. is RET faster than therapist mediated interventions, which children could benefit most from RET interventions and under which conditions. As a final clinical conclusion, and supported by the data obtained during the experiments, we can say that RET is a promising approach that could be as efficient as (or even more efficient than) classical interventions for a large variety of outcomes in the case of children with ASD.

Given the technical requirements of this project, unfortunately, very few end-users might benefit from the system developed in this project. For that reason, a simplified version of the DREAM system has been implemented as one of the Ask $\mathrm{NAO}^{4}$ Tablet applications (Figure 9). Its functionality is as follows. The caregiver uses the application as an administrator (which allows him/her to monitor NAO's activities and to ac-

\footnotetext{
${ }^{4}$ https : //asknao.aldebaran.com/
} 
cess the control panel), and the child uses another tablet, that can only interact with the information that NAO sends. This way, the caregiver never has to physically move away from the child and the robot to set up activities. This solution facilitates the ability of the caregiver, being an observer, to retrieve answers and send encouragement messages, while the robot is interacting with the child. Currently, thirteen Ask NAO Tablet applications have been developed in the DREAM project. These applications will be tested with children with ASD following the testing protocol created by our therapists. Results and feedback from therapists after this testing will be used to update the Ask NAO Tablet applications.

\section{Acknowledgment}

The work leading to these results has received funding from the European Commission 7th Framework Program as a part of the DREAM project, grant no. 611391. The Authors obtained consent for the use of all the photos in this publication.

\section{References}

[1] American Psychiatric Association, Diagnostic and Statistical Manual of Mental Disorders (DSM-5®). American Psychiatric Pub, 2013.

[2] S. Thill, C. A. Pop, T. Belpaeme, T. Ziemke, and B. Vanderborght, "Robot-assisted therapy for autism spectrum disorders with (partially) autonomous control: Challenges and outlook," Paladyn, vol. 3, no. 4, pp. 209-217, 2012.

[3] P. G. Esteban, P. Baxter, T. Belpaeme, E. Billing, H. Cai, H.-L. Cao, M. Coeckelbergh, C. Costescu, D. David, A. De Beir et al., "How to build a supervised autonomous system for robot-enhanced therapy for children with autism spectrum disorder," Paladyn Journal of Behavioral Robotics, vol. 8, no. 1, pp. 18-38, 2017.

[4] J. J. Diehl, L. M. Schmitt, M. Villano, and C. R. Crowell, "The clinical use of robots for individuals with autism spectrum disorders: A critical review," Research in Autism Spectrum Disorders, vol. 6, no. 1, pp. 249-262, 2012.

[5] B. Robins, K. Dautenhahn, R. Te Boekhorst, and A. Billard, "Robotic assistants in therapy and education of children with autism: can a small humanoid robot help encourage social interaction skills?" Universal Access in the Information Society, vol. 4, no. 2, pp. 105-120, 2005.

[6] F. Sartorato, L. Przybylowski, and D. K. Sarko, "Improving therapeutic outcomes in autism spectrum disorders: Enhancing social communication and sensory processing through the use of interactive robots," Journal of psychiatric research, vol. 90, pp. 1-11, 2017.

[7] C. A. Huijnen, M. A. Lexis, R. Jansens, and L. P. de Witte, "Mapping robots to therapy and educational objectives for children with autism spectrum disorder," Journal of Autism and Developmental Disorders, vol. 46, no. 6, pp. 2100-2114, 2016.

[8] B. Scassellati, H. Admoni, and M. Matarić, "Robots for use in autism research," Annual Review of Biomedical Engineering, vol. 14, pp. 275-294, 2012.

[9] M. Coeckelbergh, C. Pop, R. Simut, A. Peca, S. Pintea, D. David, and B. Vanderborght, "A survey of expectations about the role of robots in robotassisted therapy for children with asd: Ethical acceptability, trust, sociability, appearance, and attachment," Science and Engineering Ethics, vol. 22, no. 1, pp. 47-65, 2016.

[10] A. Peca, "Robot enhanced therapy for children with autism disorders: Measuring ethical acceptability," IEEE Technology and Society Magazine, vol. 35, no. 2, pp. 54-66, 2016.

[11] H.-L. Cao, P. G. Esteban, A. De Beir, R. Simut, G. van de Perre, D. Lefeber, and B. Vanderborght, "A survey on behavior control architectures for social robots in healthcare interventions," International Journal of Humanoid Robotics, vol. 14, no. 04, p. 1750021, 2017.

[12] H.-L. Cao, G. van de Perre, J. Kennedy, E. Senft, P. G. Esteban, A. De Beir, R. Simut, T. Belpaeme, D. Lefeber, and B. Vanderborght, "A personalized and platform-independent behavior control system for social robots in therapy: development and applications," IEEE Transactions on Cognitive and Developmental Systems, 2018.

[13] X. Zhou, H. Cai, Y. Li, and H. Liu, "Two-eye modelbased gaze estimation from a kinect sensor," in 2017 IEEE International Conference on Robotics and Automation (ICRA). Marina Bay Sands, Singapore: IEEE, 2017, pp. 1646-1653.

[14] B. Liu, H. Yu, X. Zhou, D. Tang, and H. Liu, "Combining 3D joints moving trend and geometry property for human action recognition," in 2016 IEEE International Conference on Systems, Man, and Cybernetics (SMC). Budapest, Hungary: IEEE, 2016, pp. 332-337.

[15] Y. Wang, H. Yu, B. Stevens, and H. Liu, "Dynamic facial expression recognition using local patch and lbp-top," in 8th International Conference on Human System Interactions (HSI). Warsaw, Poland: IEEE, 2015, pp. 362-367. 
[16] X. Zhou, H. Yu, H. Liu, and Y. Li, "Tracking multiple video targets with an improved GM-PHD tracker," Sensors, vol. 15, no. 12, pp. $30240-$ $30260,2015$.

[17] T. Kinnunen and H. Li, "An overview of textindependent speaker recognition: From features to supervectors," Speech Communication, vol. 52, no. 1, pp. 12-40, 2010.

[18] D. Wall, J. Kosmicki, T. Deluca, E. Harstad, and V. Fusaro, "Use of machine learning to shorten observation-based screening and diagnosis of autism," Translational psychiatry, vol. 2, no. 4, p. e100, 2012.

[19] M. Bartlett, T. Belpaeme, and S. Thill, "Towards a full spectrum diagnosis of autistic behaviours using human robot interactions," in 1st workshop on Social Robots in therapy: focusing on autonomy and Ethical Challenges (SREC'18), Chicago, IL, USA, 2018.

[20] P. G. Esteban, H.-L. Cao, A. De Beir, G. Van de Perre, D. Lefeber, and B. Vanderborght, "A multilayer reactive system for robots interacting with children with autism," in 5th International Symposium on New Frontiers in Human-Robot Interaction. Shelfield, UK: AIBS, 2016, pp. 1-4.

[21] E. Senft, P. Baxter, J. Kennedy, S. Lemaignan, and T. Belpaeme, "Supervised autonomy for online learning in human-robot interaction," Pattern Recognition Letters, vol. 99, pp. 77-86, 2017.

[22] C. Lord, S. Risi, L. Lambrecht, E. H. Cook, B. L. Leventhal, P. C. DiLavore, A. Pickles, and M. Rutter, "The autism diagnostic observation schedule-generic: A standard measure of social and communication deficits associated with the spectrum of autism," Journal of Autism and Developmental Disorders, vol. 30, no. 3, pp. 205-223, 2000.

[23] P. Baxter, R. Wood, and T. Belpaeme, "A touchscreen-based 'sandtray' to facilitate, mediate and contextualise human-robot social interaction," in 7th Annual ACM/IEEE International Conference on Human-Robot Interaction (HRI). Boston, Massachusetts, USA: ACM, 2012, pp. 105-106.

[24] D. O. David, C. A. Costescu, S. Matu, A. Szentagotai, and A. Dobrean, "Developing joint attention for children with autism in robot-enhanced therapy," International Journal of Social Robotics, vol. 10, no. 5, pp. 595-605, 2018.

[25] G. Veruggio and F. Operto, "Roboethics: Social and ethical implications of robotics," in Springer Handbook of Robotics, B. Siciliano and O. Khatib, Eds. Berlin: Springer, 2008, pp. 1499-1524.

[26] K. Richardson, M. Coeckelbergh, K. Wakunuma, E. Billing, T. Ziemke, P. Gomez, B. Vanderborght, and T. Belpaeme, "Robot enhanced therapy for children with autism (DREAM): A social model of autism," IEEE Technology and Society Magazine, vol. 37, no. 1, pp. 30-39, 2018.

[27] C. A. Costescu, B. Vanderborght, and D. O. David, "Beliefs, emotions, and behaviors-differences between children with asd and typically developing children. a robot-enhanced task," Journal of Evidence-Based Psychotherapies, vol. 16, no. 2, p. 221, 2016. 